\title{
Treatment of Varicocele in Children and Adolescents: A Systematic Review and Meta-analysis from the European Association of Urology/European Society for Paediatric Urology Guidelines Panel
}

\author{
Mesrur Selcuk Silay ${ }^{a, *}$, Lisette Hoen $^{b}$, Josine Quadackaers ${ }^{c}$, Shabnam Undre $^{d}$, Guy Bogaert ${ }^{e}$, \\ Hasan Serkan Dogan ${ }^{f}$, Radim Kocvara $^{g}$, Rien J.M. Nijman ${ }^{c}$, Christian Radmayr $^{h}$, Serdar Tekgul $^{f}$, \\ Raimund Stein ${ }^{i}$ \\ ${ }^{a}$ Division of Pediatric Urology, Department of Urology, Istanbul Medeniyet University, Istanbul, Turkey; ${ }^{\mathrm{b}}$ Department of Urology, Erasmus MC, Rotterdam, \\ The Netherlands; ${ }^{\mathrm{c}}$ Department of Urology and Pediatric Urology, University Medical Centre Groningen, Groningen, The Netherlands; ${ }^{\mathrm{d}}$ Department of Pediatric \\ and Adult Urology, East and North Herts NHS Trust, Stevenage, UK; ${ }^{\mathrm{e}}$ Department of Urology, University of Leuven, Leuven, Belgium; ${ }^{\mathrm{f}}$ Division of Pediatric \\ Urology, Department of Urology, Hacettepe University, Ankara, Turkey; ${ }^{\mathrm{g}}$ Department of Urology, General Teaching Hospital and Charles University 1 st Faculty of \\ Medicine in Praha, Prague, Czech Republic; ${ }^{\mathrm{h}}$ Department of Urology, Medical University of Innsbruck, Innsbruck, Austria; ${ }^{\mathrm{i}}$ Department of Pediatric, Adolescent \\ and Reconstructive Urology, University of Medical Center Mannheim, Medical Faculty Mannheim, Heidelberg University, Mannheim, Germany
}

\section{Article info}

Article history:

Accepted September 24, 2018

Associate Editor:

James Catto

\section{Keywords:}

Varicocele

Children

Adolescent

Recurrence

Hydrocele

Paternity

\begin{abstract}
Context: The benefits and harms of intervention (surgical or radiological) versus observation in children and adolescents with varicocele are controversial.

Objective: To systematically evaluate the evidence regarding the short- and long-term outcomes of varicocele treatment in children and adolescents.

Evidence acquisition: A systematic review and meta-analysis was conducted in accordance with the Preferred Reporting Items for Systematic Reviews and Meta-analysis (PRISMA) statement. A priori protocol was registered to PROSPERO (CRD42018084871), and a literature search was performed for all relevant publications published from January 1980 until June 2017. Randomized controlled trials (RCTs), nonrandomized comparative studies (NRSs), and single-arm case series including a minimum of 50 participants were eligible for inclusion.

Evidence synthesis: Of 1550 articles identified, 98 articles including 16130 patients (7-21 yr old) were eligible for inclusion (12 RCTs, 47 NRSs, and 39 case series). Varicocele treatment improved testicular volume (mean difference $1.52 \mathrm{ml}, 95 \%$ confidence interval [CI] 0.73-2.31) and increased total sperm concentration (mean difference $25.54,95 \%$ CI 12.84-38.25) when compared with observation. Open surgery and laparoscopy may have similar treatment success. A significant decrease in hydrocele formation was observed in lymphatic sparing versus non-lymphatic sparing surgery $(p=0.02)$. Our findings are limited by the heterogeneity of the published data, and a lack of long-term outcomes demonstrating sperm parameters and paternity rates.

Conclusions: Moderate evidence exists on the benefits of varicocele treatment in children and adolescents in terms of testicular volume and sperm concentration. Current evidence does not demonstrate superiority of any of the surgical/interventional techniques regarding treatment success. Long-term outcomes including paternity and fertility still remain unknown.

Patient summary: In this paper, we review benefits and harms of varicocele treatment in children and adolescents. We found moderate evidence that varicocele treatment results in improvement of testicular volume and sperm concentration. Lymphatic sparing surgery decreases hydrocele formation. Paternity and fertility outcomes are not clear.
\end{abstract}

(c) 2018 European Association of Urology. Published by Elsevier B.V. All rights reserved.

\footnotetext{
* Corresponding author. Division of Pediatric Urology, Department of Urology, Istanbul Medeniyet University, Doktor Erkin Caddesi, 34722, Kadikoy, Istanbul, Turkey. Tel. +90 505 6454005; Fax: +90 2124530453.

E-mail address: selcuksilay@gmail.com (M.S. Silay).
}

https://doi.org/10.1016/j.eururo.2018.09.042

0302-2838/@ 2018 European Association of Urology. Published by Elsevier B.V. All rights reserved. 


\section{Introduction}

Varicocele in children and adolescents is one of the most controversial topics in pediatric urology and has an incidence of up to $14-20 \%$ in adolescent boys, similar to adults [1]. It is defined as the abnormal dilatation of the veins within the pampiniform plexus as a result of venous reflux. It is believed that varicocele may cause fertility problems by negatively affecting sperm parameters, including density, motility, and morphology, and by decreasing testicular volume [2,3]. In a recent meta-analysis (MA), it has been demonstrated that treatment of adolescent varicocele positively affects sperm parameters and may lead to increased testicular volume, which is also known as "testicular catch-up growth" [4].

On the contrary, there are several limitations of the previously published MA. Publications by both Nork et al. [2] and Zhou et al. [3] included nonrandomized comparative studies (NRSs) in the statistical analysis, which have limited their level of evidence. In addition, both of them focus mainly on semen parameters and testicular volume, while the outcomes of the treatment (success, recurrence, complications, etc.) have not been reported. A recent MA by Locke et al. [4] overcame this limitation by analyzing only randomized controlled trials (RCTs) to elucidate some points over varicocele treatment. However, this study was also limited by the lack of information on items such as comparison of the surgical techniques, surgical success, hydrocele formation, complication rates, and paternity in the long term.

With this comprehensive systematic review (SR) and MA, we aim to determine the benefits and harms of varicocele intervention in pediatrics in the short and long term for the various interventions available.

\section{Evidence acquisition}

\subsection{Search strategy}

This study was conducted in accordance with the Preferred Reporting Items for Systematic Reviews and Meta-analysis (PRISMA) statement. An a priori protocol was registered at the PROSPERO database (reference CRD42018084871). The literature search was performed for all relevant publications published from January 1980 until June 2017, using the following databases: Embase, MEDLINE, Cochrane SRs, Cochrane Central, Cochrane HTA, Chinicaltrial.gov, and WHO International Clinical Trials Registry Platform Search Portal. We used the string terms varicocele AND pediatrics or synonyms of this. Publications included were original articles regarding interventions for varicocele in the English language. All abstracts and following relevant full texts were evaluated by two of the four independent reviewers (M.S.S., S.U., J.S.L.T.Q and L.A.H.). Disagreements were resolved by interactive discussion. A complete search strategy is provided in the Supplementary material.

\subsection{Types of study designs}

RCTs, NRSs, and single-arm case series including a minimum of 50 participants were eligible for inclusion. SRs and narrative reviews were excluded, but were used as a source for the discussion.

\subsection{Types of participants}

Only children and adolescents $\leq 21 \mathrm{yr}$ of age, with a clinically diagnosed varicocele, were included, regardless of the grade of severity (eg, Dubin-Amelar classification: grades 1-3). Our exclusion criteria were subclinical diagnosis, secondary varicocele, previous inguinoscrotal surgery on either side, any local or systemic treatment that can affect fertility, testicular trauma, previous orchitis, and endocrinological or chromosomal syndromes. If studies did not mention these characteristics, we assumed them not to be present.

\subsection{Types of interventions}

Intervention was divided into surgical (all levels including Palomo, Ivanissevich, subinguinal, with or without microscope or loupe, laparoscopy) and radiological (eg, sclerotherapy, embolization, antegrade versus retrograde) interventions. These interventions were compared with control groups without intervention (including observation) or no control in the single-arm case series.

\subsection{Types of outcome measures}

The primary outcome for benefits were short-term cure or success (defined as resolution of varicocele) measured $<9$ mo, also including downgrading in Dubin-Amelar grade. The primary outcome for harms were interventional complication, such as, but not limited, to testicular atrophy, secondary hydrocele, wound infection, conversion rate, etc.

The secondary outcomes for benefits were testicular catch-up growth, pain resolution, sperm parameters, paternity, and hormonal status (changes on folliclestimulating hormone [FSH], luteinizing hormone [LH], and testosterone) at $>12$ mo follow-up or as defined by trialists. The definition of "catch-up growth" was not uniform among the included studies. In general, for patients with varicocele and considerable testicular size discrepancy, "catch-up growth" means that the size of the left testis caught up with the right testis after any type of intervention or observation. The secondary harms included failure rate, delayed hydrocele, and delayed testicular atrophy.

\subsection{Assessment of risk of bias}

The risk of bias was assessed for each included study by the four review authors independently. Any disagreements were resolved by discussion. Risk of bias for RCTs was judged by using the recommended tool in the Cochrane Handbook for Systematic Reviews of Interventions. This included the assessment of random sequence generation, allocation concealment, blinding of participants and personnel, blinding of outcome assessment, incomplete outcome data, selective reporting, and other sources of bias. 


\subsection{Data analysis}

The number or percent of treatment success, recurrence and complications, and other outcomes measured including testicular volumes and pain resolution were extracted from the eligible studies. Long-term outcomes including sperm parameters, hormonal changes, and paternity rates were also extracted.

MAs were performed only for the outcomes derived from RCTs. This was applicable for the following parameters: changes in testicular volume and sperm parameters during the comparison of intervention versus observation. Hydrocele development after lymphatic sparing versus nonsparing surgery was also meta-analyzed.

MAs were intended for the other comparisons including paternity, pain resolution, and types of surgeries. However, due to the lack of this evidence in RCTs, some of the additional data from NRSs have been represented in forest plots without MA (due to methodological heterogeneity and the high risk of bias).

Regarding the binary/dichotomous/categorical benefit or harm outcomes, odds ratios (ORs) were used where available. Mean difference (MD) with 95\% confidence intervals (CIs) were used to report continuous outcomes.

\section{Evidence synthesis}

\subsection{Quantity of evidence identified}

The search and selection process of the articles are demonstrated in the PRISMA flow diagram (Fig. 1). A total of 1550 abstracts and titles were screened and 160 were retrieved for full-text screening. Finally, 98 studies were found eligible, recruiting a total of 16130 children and adolescents (RCTs: 1605, NRSs: 9672, case series: 4853). This included 12 RCTs [5-16], 47 NRSs [17-63], and 39 case series [64-102].

\section{2. $\quad$ Characteristics of the included studies}

Baseline characteristics of the 12 RCTs are presented in Table 1. Owing to the high numbers of included studies, baseline characteristics of the NRSs and case series are not demonstrated in tables, but instead the highlights are reported below.

\subsubsection{Characteristics of RCTs}

The comparison parameters for the included RCTs were variable and listed in Table 1. Four studies randomized observation with different types of interventions $[5,9,10,14]$. The rest of the papers compared different intervention techniques between each other. Only one study included embolization with observation [5].

Although all recruited patients had clinical varicocele, the grades were variable. The majority of the studies included GII and GIII varicocele, whereas three papers included GI varicocele $[10,14,16]$ and two papers did not report the grades $[9,13]$.
The numbers of the included patients, mean follow-up periods, and inclusion and exclusion criteria are also listed in Table 1.

\subsubsection{Characteristics of NRSs}

A total of 47 NRSs (seven prospective and 40 retrospective) including 9672 patients met the inclusion criteria. The comparison parameters were variable, including open versus laparoscopy [21-23,30,32,34,38,41,42,46,51,62,63], laparoscopy or open surgery versus sclerotherapy/embolization [18,30,54-56], laparoscopic or open artery sparing versus non-artery sparing [25,29,42,43,52,56,59,61,62], and lymphatic sparing versus nonsparing $[27,36,49,60]$.

The grades of varicocele were 2 and 3 in the majority of the studies, whereas the grades were not reported in 19 of the studies. Indications for surgery were variable and were also reported in 12 of the studies.

\subsubsection{Characteristics of case series}

A total of 38 case series (four prospective and 34 retrospective) including 4853 patients met the inclusion criteria. Sixteen studies reported the outcomes of laparoscopic varicocelectomies

$[64,66,67,72,74,75,77,80,81,83,85,86,89,90,94,96]$. Eleven studies reported outcomes of sclerotherapy/embolization $[69,71,76,78,82,87,92,93,97,99,102]$. The remainder reported outcomes of various types of open surgery $[65,68,70,73,79,84,88,91,95,98,100,101]$.

The grades of varicocele were again 2 and 3 in the majority of the studies, whereas the grade was not reported in nine of the studies. In one study reporting the outcomes of antegrade scrotal sclerotherapy, the authors also included 12 subclinical varicoceles in their study [69]. Indications for surgery/intervention were variable, including grade 3 varicocele, hypotrophy, presence of symptoms, and abnormal semen analysis as defined by the trialists.

\subsection{Risk of bias summary for the included studies}

Figure 2 demonstrates the risk of bias summary and confounding assessments for the 12 RCTs. A low risk of selection bias was present for the majority of the studies, whereas a high risk was present only for one study [15]. A high risk of performance bias and an unclear risk of detection bias were present for all included RCTs. Attrition bias and reporting bias were at low risk in general.

\subsection{Outcomes of included studies}

\subsubsection{Data from RCTs}

The outcome results of 12 RCTs are summarized and demonstrated in Table 2.

3.4.1.1. Treatment success. The definition of success in 12 of the RCTs was variable and included disappearance of varicocele, testicular catch-up growth, and improvement in semen analysis. Success rates (disappearance of varicocele) were between $87 \%$ and $100 \%$. It was not reported in three of the included studies $[9,10,14]$. 


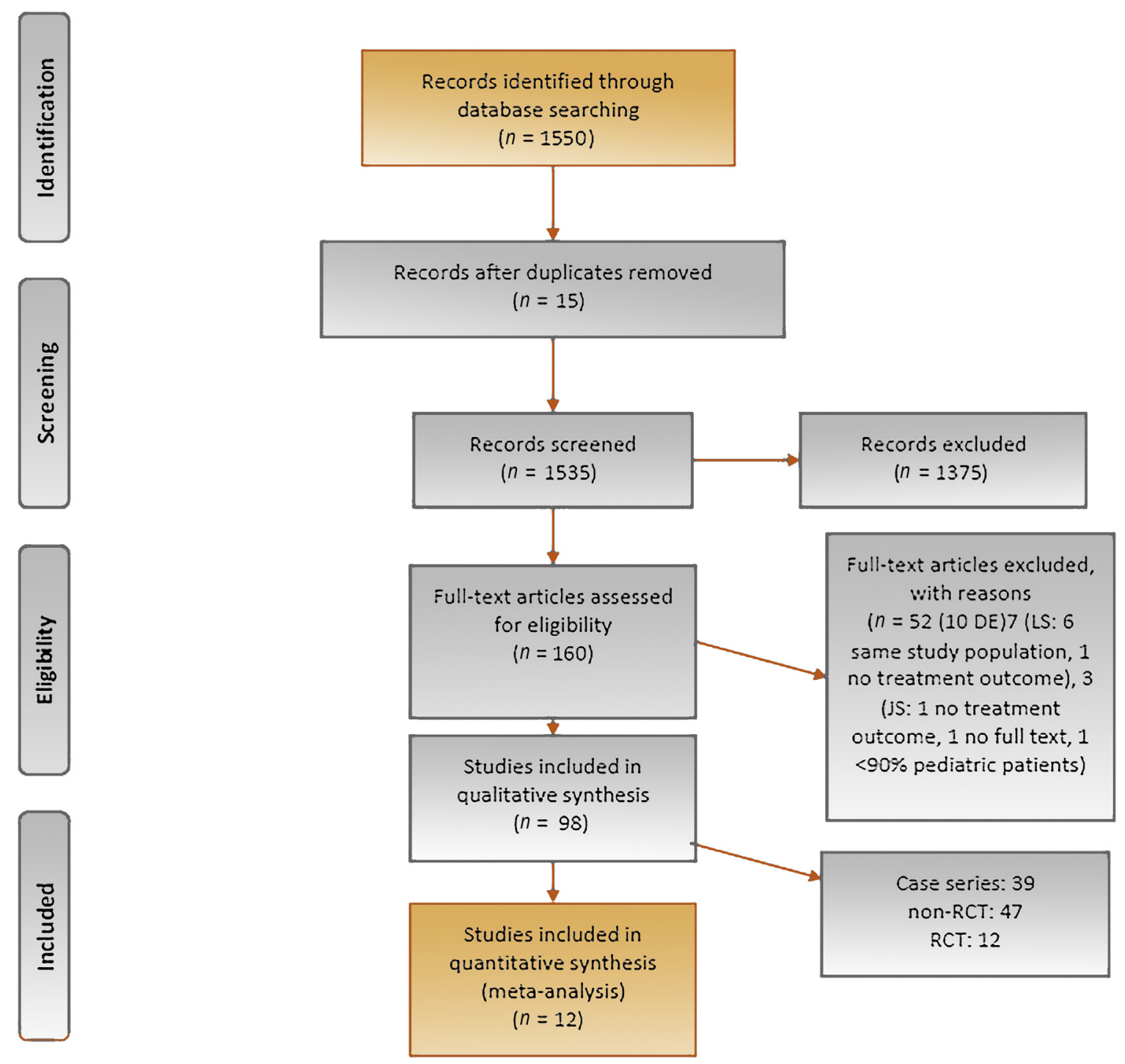

Fig. 1 - PRISMA flow diagram. PRISMA = Preferred Reporting Items for Systematic Reviews and Meta-analysis; RCT = randomized controlled trial.

Podkamenev et al. [6] randomized open ( $n: 220)$ versus laparoscopic ( $n: 434)$ treatment of varicocele. In both groups, the Palomo technique was performed. After a mean follow-up of $6 \mathrm{mo}$, the success rates were similar among the groups (open: $217 / 220,98.6 \%$; laparoscopic varicocelectomy: 428/434, 98.6\%; $p>0.05$ ).

Shiraishi et al. [12] randomized the levels of microsurgical varicocelectomy performed in open fashion. They compared the outcomes of subinguinal ( $n: 41)$ versus high inguinal ( $n$ : 40) varicocelectomy in children. After a mean follow-up period of 38.2 mo (range: $24-85 \mathrm{mo}$ ) there were no recurrences in any of the groups, and the overall success rate was $100 \%$.

3.4.1.2. Complication of surgery/interventions. The complications recorded from the available RCTs included hydrocele, atrophy, and any other complication detected by the trialists. The most common complication reported was hydrocele. In four RCTs, complications were not reported $[5,9,14,16]$.

The rate of hydrocele formation following varicocelectomy was $0-12 \%$ with 6-85 mo of follow-up, and was lowest when magnification (either loupe or microscope) was performed [9,13] and seemed to be significantly higher after open Palomo surgery [10].

The MA of two RCTs that compared lymphatic sparing versus nonsparing surgery is demonstrated in Figure 3 [7,11]. Lymphatic sparing significantly decreased hydrocele rates $(p=0.02$ ) and the odds ratio (OR) was 0.08 (95\% CI 0.01, $0.67)$.

3.4.1.3. Testicular volume changes and catch-up growth. Testicular volume measurements (either by ultrasound or by orchidometer) and/or catch-up growth rates were recorded in eight of the RCTs [5,8-10,12-14,16], whereas it was not reported in the remaining four RCTs $[6,7,11,15]$. 
Table 1 - Summary of findings-baseline characteristics of RCTs

\begin{tabular}{|c|c|c|c|c|c|c|c|}
\hline $\begin{array}{l}\text { Study ID (year), } \\
\text { recruitment period }\end{array}$ & $N$ & $\begin{array}{l}\text { Age }(\mathrm{yr}), \text { mean }(\mathrm{SD}) \\
\text { median (range) }\end{array}$ & Comparison parameters & $\begin{array}{l}\text { Grade severity of } \\
\text { varicocele }\end{array}$ & Inclusion criteria & Exclusion criteria & $\begin{array}{l}\text { Indication for surgery or } \\
\text { intervention }\end{array}$ \\
\hline $\begin{array}{l}\text { Laven et al (1992) [5], } \\
\text { NR }\end{array}$ & 67 & $17-20$ & $\begin{array}{l}\text { Observation vs } \\
\text { embolization }\end{array}$ & $\begin{array}{l}\text { II: } 10 \\
\text { III: } 43\end{array}$ & $\begin{array}{l}\text { Adolescents with } \\
\text { varicocele }\end{array}$ & NR & NR \\
\hline $\begin{array}{l}\text { Podkamenev et al } \\
\text { (2002) [6], 1995- } \\
2000\end{array}$ & 654 & $7-17$ & $\begin{array}{l}\text { Open vs laparoscopic } \\
\text { varicocelectomy }\end{array}$ & II and III & Left VC & Refusal of the study & NR \\
\hline $\begin{array}{l}\text { Golebiewski et al } \\
\text { (2007) [7], 2003- } \\
2005\end{array}$ & 52 & $12-16(14.7)$ & $\begin{array}{l}\text { Laparoscopic lymphatic } \\
\text { nonsparing vs sparing }\end{array}$ & All patients GIII & $\begin{array}{l}\text { Unilateral G3 } \\
\text { varicocele }\end{array}$ & NR & $\begin{array}{l}\text { G3 varicocele, asymmetric } \\
\text { testicular growth and } \\
\text { infrequent discomfort }\end{array}$ \\
\hline $\begin{array}{l}\text { Marte et al (2014) [8], } \\
\text { 2011-2013 }\end{array}$ & 69 & $14.5(11-17)$ & $\begin{array}{l}\text { Single-incision } \\
\text { laparoscopic } \\
\text { varicocelectomy vs } \\
\text { conventional laparoscopic } \\
\text { varicocelectomy }\end{array}$ & II and III & $\begin{array}{l}\text { G2-3 varicocele or } \\
\text { ipsilateral testicular } \\
\text { hydrotrophy }\end{array}$ & $\begin{array}{l}\text { History of previous abdominal } \\
\text { surgery }\end{array}$ & G2-3 VC or hypotrophy \\
\hline $\begin{array}{l}\text { Moursy et al (2013) } \\
\text { [9], 2004-2007 }\end{array}$ & 173 & $14.3(12-16)$ & $\begin{array}{l}\text { Inguinal VC using loupe vs } \\
\text { no treatment }\end{array}$ & NR & NR & NR & Hypotrophy >20\% \\
\hline $\begin{array}{l}\text { Paduch and } \\
\text { Niedzielski (1997) } \\
\text { [10], NR }\end{array}$ & 124 & $\begin{array}{l}15-19 \\
\text { Treated: } 16.7 \\
\text { Untreated: } 16.5\end{array}$ & $\begin{array}{l}\text { Palomo artery sparing vs } \\
\text { no treatment }\end{array}$ & $\begin{array}{l}\text { GI: } 7 \\
\text { GII: } 48 \\
\text { GIII: } 59\end{array}$ & Clinically detected VC & NR & NR \\
\hline $\begin{array}{l}\text { Schwentner et al } \\
\text { (2006) [11], NR }\end{array}$ & 50 & $\begin{array}{l}\text { Group1: } 17.8 \\
\text { Group2: } 16.5\end{array}$ & $\begin{array}{l}\text { Laparoscopic VC w/wo } \\
\text { isosulfan blue }\end{array}$ & $\begin{array}{l}\text { GIII: } 27 \\
\text { GII: } 23\end{array}$ & $\mathrm{NR}>$ & $\begin{array}{l}\text { Previous groin surgery, } \\
\text { secondary procedures, hernia } \\
\text { and hydroceles, history of } \\
\text { undescended testis }\end{array}$ & $\begin{array}{l}\text { Low sperm counts (in young } \\
\text { men), scrotal pain and } \\
\text { testicular atrophy or severe } \\
\text { cosmetic impairment (grade } \\
\text { III) }\end{array}$ \\
\hline $\begin{array}{l}\text { Shiraishi et al (2016) } \\
\text { [12], 2008-2014 }\end{array}$ & 81 & $13.4(9-18)$ & $\begin{array}{l}\text { Subinguinal vs high } \\
\text { inguinal VC (both } \\
\text { microsurgical) }\end{array}$ & G2-3 & $\begin{array}{l}\text { G } 2-3 \text { VC w pain }(20 \% \\
\text { subinguinal and } 25 \% \\
\text { high) or asymmetry of } \\
\geq 20 \%\end{array}$ & $\begin{array}{l}\text { Prior surgery, older than } 18, \mathrm{GI} \\
\text { VC, bilat VC solitary testis }\end{array}$ & Pain or asymmetry \\
\hline $\begin{array}{l}\text { Spinelli et al (2016) } \\
\text { [13], 2008-2013 }\end{array}$ & 70 & $14.5(7-17)$ & $\begin{array}{l}\text { Microsurgical artery and } \\
\text { lymph sparing inguinal: } \\
\text { with vs without delivery of } \\
\text { testis }\end{array}$ & NR & $\begin{array}{l}\text { Unilateral varicocele } \\
\text { with testicular volume } \\
\text { discrepancy }>20 \%\end{array}$ & $\begin{array}{l}\text { Previous groin surgery, } \\
\text { endocrine or multisystem } \\
\text { anomaly affecting testis } \\
\text { volume }\end{array}$ & $\begin{array}{l}\text { Unilateral varicocele with } \\
\text { testicular volume } \\
\text { discrepancy }>20 \%\end{array}$ \\
\hline $\begin{array}{l}\text { Yamamoto et al } \\
\text { (1995) [14], NR }\end{array}$ & $51+18$ & $\begin{array}{l}\text { Untreated: } 18.4 \\
\text { Treated: } 18.6 \\
\text { Control: } 18.2\end{array}$ & $\begin{array}{l}\text { High inguinal VC vs } \\
\text { observation vs control }\end{array}$ & $\begin{array}{l}\text { G1: } 20 \\
\text { G2: } 29 \\
\text { G3: } 2\end{array}$ & NR & NR & NR \\
\hline $\begin{array}{l}\text { Zampieri et al (2007) } \\
{[15], 1999-2003}\end{array}$ & 122 & $14.3(12-16)$ & $\begin{array}{l}\mathrm{LV} w \text { artery sparing vs } \\
\text { nonsparing }\end{array}$ & $\begin{array}{l}\text { G2: } 82 \\
\text { G3: } 40\end{array}$ & Idiopathic VC & NR & Hypotrophy with varicocele \\
\hline $\begin{array}{l}\text { Mohseni et al (2011) } \\
{[16], 2006-2009}\end{array}$ & 74 & $13(8-18)$ & $\begin{array}{l}\text { Retroperitoneal vs inguinal } \\
\text { (only for shunt-type VC) }\end{array}$ & $\begin{array}{l}\text { G1: } 9 \\
\text { G2: } 35 \\
\text { G3: } 30\end{array}$ & $\begin{array}{l}\text { Shunt and stop type VC } \\
\text { with testicular } \\
\text { asymmetry } \geq 20 \%\end{array}$ & NR & $\begin{array}{l}\text { Testicular asymmetry } \\
\text { (difference } \geq 20 \% \text { ) }\end{array}$ \\
\hline
\end{tabular}

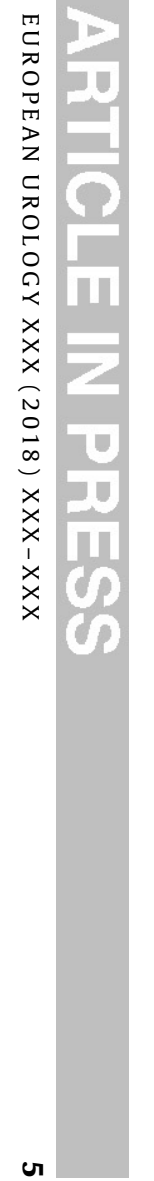




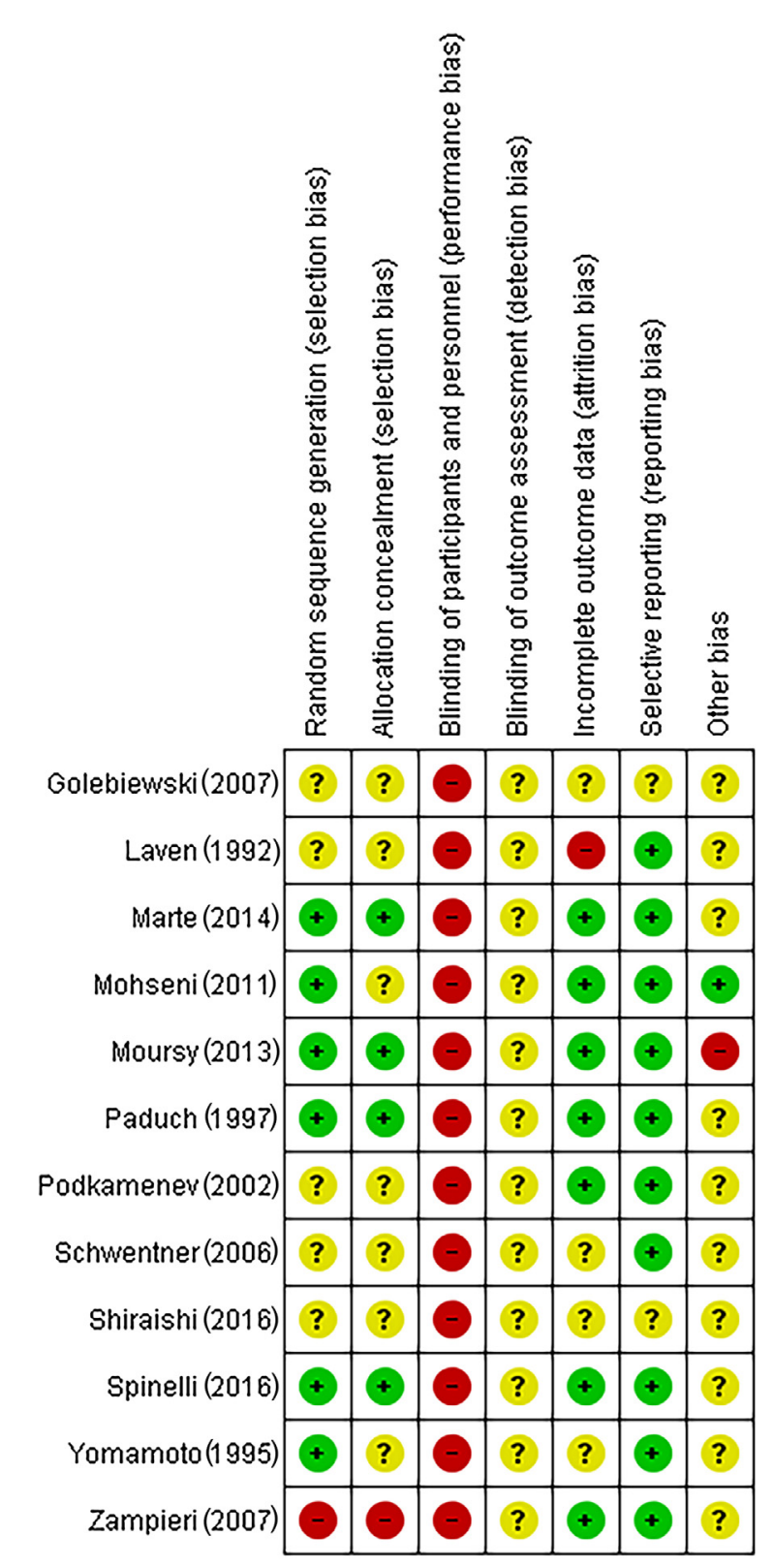

Fig. 2 - Risk of bias summary for 12 RCTs. RCT = randomized controlled trial.

Four studies were included in an MA all of which compared surgery/intervention versus observation in children with varicocele (Fig. 4). Among them, only Laven et al. [5] compared the outcomes of embolization versus observation, whereas the remaining three studies compared varicocelectomy (inguinal and high inguinal) versus observation $[9,10,14]$. Testicular volumes were significantly higher in the treated group versus the nontreated group $(p<0.001)$ and the OR was 1.52 (95\% CI 0.73, 2.31).

3.4.1.4. Changes in sperm parameters. Sperm parameters were recorded in three of the RCTs [5,14,15], whereas in one study it was mentioned without providing any data [9]. Although Tanner stages of the patients were not reported in the studies, semen samples were taken from two of them after they had become 18 yr old [9,15]; by contrast, samples were taken from patients aged between 17 and $21 \mathrm{yr}$ in the study of Laven et al. [5] and from those 15-21 yr old in the study of Yamamoto et al. [14]. In the remaining eight RCTs, sperm parameters were not reported.

Two RCTs reported pre- and postoperative spermiogram values in both treated and untreated groups [5,14]. These two studies underwent an MA (Fig. 5). It was found that sperm concentration was significantly higher in the treated groups versus the nontreated groups, with a mean difference of $25.54 \mathrm{million} / \mathrm{ml}(p<0.001)$. However motility (MD: $2.80 \%, 95 \%$ CI 2.39-7.99, $p=0.29$ ) and morphology (MD:-1.99\%, 95\% CI-7.21 to 3.23, $p=0.46$ ) parameters were comparable between treated and untreated groups.

3.4.1.5. Resolution of pain. Resolution or recurrence of pain after treatment of varicocele was mentioned in only two of the RCTs [11,12]. Schwentner et al. [11] reported outcomes of laparoscopic varicocelectomy with and without staining for lymphatic vessels. The total number of patients with preoperative pain is not reported. However, at the end of follow-up, there were two patients with persistent pain in both groups and in one of them the pain resolved spontaneously. In another study by Shiraishi et al. [12], scrotal pain associated with varicocele diminished in all children $(24 / 24,100 \%)$ postoperatively. In the same study, three patients without preoperative pain reported postoperative pain, which resolved within 3-6 mo of follow-up.

3.4.1.6. Hormonal status. Only two RCTs reported the values of the hormonal status (serum LH, FSH, and testosterone) of the children postoperatively [9,14]. In the study by Moursy et al. [9], all FSH levels were normal at follow-up, except one patient in group B2 (observation) who required surgical correction because of elevated FSH levels and oligoasthenospermia, which normalized afterward. In the study by Yamamoto et al. [14], hormone measurements were performed at intake and after $1 \mathrm{yr}$ of follow-up. Serum LH, FSH, and testosterone levels were determined. During intake and after $1 \mathrm{yr}$ of follow-up, all hormone levels were within normal range in both treated and untreated groups.

3.4.1.7. Paternity rates. Paternity rates were not recorded in any of the RCTs included in this study.

\subsubsection{Data from NRSs and case series}

The outcome results of 47 NRSs and 39 case series are summarized below.

3.4.2.1. Treatment success. The definition of success was highly variable in the included NRSs and case series, and included disappearance of varicocele, paternity, testicular catch-up growth, etc. On the contrary, it was not defined in 24 of 47 NRSs and in 18 of 39 case series.

The treatment success rates (disappearance of varicocele) were between $88.2 \%$ and $100 \%$ in the included NRSs, whereas the rates were between $85.1 \%$ and $100 \%$ in case series. 
Table 2 - Summary of findings-outcomes of RCTs

\begin{tabular}{|c|c|c|c|c|c|c|c|c|c|}
\hline $\begin{array}{l}\text { Study ID (year) } \\
\text { recruitment period }\end{array}$ & $\begin{array}{c}\text { Duration of } \\
\text { follow-up (mo) }\end{array}$ & $\begin{array}{l}\text { Definition for } \\
\text { cure or success }\end{array}$ & $N$ or $\%$ of success & $\begin{array}{c}N \text { or \% of } \\
\text { complications }\end{array}$ & $\begin{array}{l}N \text { or \% of } \\
\text { recurrence }\end{array}$ & $\begin{array}{l}\text { Testicular volume }(\mathrm{ml}) \text { or } \\
\text { testicular catch-up } \\
\text { growth }>9 \text { mo }(n)\end{array}$ & $\begin{array}{l}N \text { of pain } \\
\text { resolution }\end{array}$ & Sperm parameters & $\begin{array}{l}\text { Authors' } \\
\text { conclusions }\end{array}$ \\
\hline $\begin{array}{l}\text { Laven et al (1992) } \\
\text { [5], NR }\end{array}$ & 12 & NR & $\begin{array}{l}\text { Treated: } 27 / 31 \\
(87 \%)\end{array}$ & NR & $4 / 31(13 \%)$ & $\begin{array}{l}\text { Preop/postop vol. } \\
\text { Measured by } \\
\text { orchidometer: } \\
\text { Untreated: } 20 / 20.3 \\
\text { Treated: } 21.6 / 24.2 \\
(p<0.001)\end{array}$ & NR & $\begin{array}{l}\text { Preop/postop sperm } \\
\text { concentration }\left(10^{6}\right): \\
\text { Untreated: } 51.5 / 46.5 \\
\text { Treated: } 47.4 / 68.9 \\
(p<0.01) \\
\text { Motility: } \\
\text { Untreated: } 52.9 / 56.5 \\
\text { Treated: } 54.3 / 59.3 \\
(p>0.05)\end{array}$ & $\begin{array}{l}\text { Although not } \\
\text { apparent in all } \\
\text { adolescents, } \\
\text { varicocele correction } \\
\text { results in increased } \\
\text { testis volume and } \\
\text { sperm concentration }\end{array}$ \\
\hline $\begin{array}{l}\text { Podkamenev et al } \\
(2002)[6], 1995- \\
2000\end{array}$ & 6 & Resolution of VC & $\begin{array}{l}\text { LV: } 428 / 434 \\
\text { OV: } 217 / 220\end{array}$ & $\begin{array}{l}\text { LV: } 6.6 \% \\
\text { OV: } 23.1 \%\end{array}$ & $\begin{array}{l}\text { LV: } 1.84 \%(8) \\
\text { OV: } 1.36 \%(3)\end{array}$ & NR & NR & NR & $\begin{array}{l}\text { Clinical efficacy of LV } \\
\text { is superior to } \\
\text { traditional open } \\
\text { surgery }\end{array}$ \\
\hline $\begin{array}{l}\text { Golebiewski et al } \\
(2007)[7], 2003- \\
2005\end{array}$ & 14 & $\begin{array}{l}\text { Resolution of VC at } \\
\text { clinical exam and } \\
\text { Doppler US }\end{array}$ & $100 \%$ & $4 / 52$ & $0 \%$ & NR & NR & NR & $\begin{array}{l}\text { The most successful } \\
\text { method of } \\
\text { prevention of } \\
\text { hydrocele during a } \\
\text { laparoscopic Palomo } \\
\text { procedure seems to } \\
\text { be intraoperative } \\
\text { lymphatic staining } \\
\text { and preservation of } \\
\text { lymphatic vessels }\end{array}$ \\
\hline $\begin{array}{l}\text { Marte et al (2014) } \\
{[8], 2011-2013}\end{array}$ & NR & $\begin{array}{l}\text { Efficacy of the } \\
\text { procedure and } \\
\text { postop outcomes }\end{array}$ & $67 / 69(97.1 \%)$ & $2 / 69$ & $2 / 69$ & $\begin{array}{l}\text { Numbers of catch-up } \\
\text { growth for hypotrophic } \\
\text { testis: } 11 / 16 \\
\text { Preop: } 15.3 \mathrm{ml} \\
\text { Postop: } 19.5 \mathrm{ml} \\
\text { (measurement method NR) }\end{array}$ & NR & NR & $\begin{array}{l}\text { SIL-V is safe and } \\
\text { effective, and allows } \\
\text { for fast and efficient } \\
\text { isolation of the } \\
\text { vascular bundle }\end{array}$ \\
\hline $\begin{array}{l}\text { Moursy et al (2013) } \\
\text { [9], 2004-2007 }\end{array}$ & $\begin{array}{l}\text { Group A: } 78 \\
\text { Group B: } 79\end{array}$ & NR & NR & NR & 4 & $\begin{array}{l}\text { Preop/postop vol. } \\
\text { Measured by US: } \\
\text { Treated: } 10.51 / 15.6 \\
\text { Untreated: } 10.62 / 15.2 \\
(p>0.05) \\
\text { Catch-up growth: } \\
\text { Treated: } 74 \% \\
\text { Untreated: } 50 \% \\
(p<0.05) \\
\text { Reduction of testicular size } \\
\text { in } 2 \text { cases in untreated } \\
\text { group }\end{array}$ & NR & $\begin{array}{l}\text { All were normal except } \\
1 \text { in the untreated } \\
\text { group }\end{array}$ & $\begin{array}{l}\text { Although adolescent } \\
\text { varicocelectomy was } \\
\text { associated with a } \\
\text { higher percentage of } \\
\text { Patients showing } \\
\text { testicular catch-up } \\
\text { growth, the mean } \\
\text { testicular volume } \\
\text { was not significantly } \\
\text { different }\end{array}$ \\
\hline $\begin{array}{l}\text { Paduch and } \\
\text { Niedzielski (1997) } \\
\text { [10], NR }\end{array}$ & 12 & NR & NR & 15 & 5 & $\begin{array}{l}\text { Preop/postop vol. } \\
\text { Measured by US: } \\
\text { Treated: } 13.6 / 16.9 \\
(p<0.001) \\
\text { Untreated: } 13.4 / 14.5\end{array}$ & NR & NR & $\begin{array}{l}\text { Repair reverses } \\
\text { hypotrophy and } \\
\text { catch-up occurs } \\
\text { within } 12 \text { mo }\end{array}$ \\
\hline $\begin{array}{l}\text { Schwentner et al } \\
\text { (2006) [11], NR }\end{array}$ & $24.4(14-36)$ & NR & $48 / 50$ (96\%) & 5 & 2 & NR & $\begin{array}{l}N: 1 / 4 \\
\text { patients }\end{array}$ & NR & $\begin{array}{l}\text { LSLV prevents } \\
\text { hydrocele and edema }\end{array}$ \\
\hline
\end{tabular}


Table 2 (Continued)

\begin{tabular}{|c|c|c|c|c|c|c|c|c|c|}
\hline $\begin{array}{l}\text { Study ID (year) } \\
\text { recruitment period }\end{array}$ & $\begin{array}{c}\text { Duration of } \\
\text { follow-up (mo) }\end{array}$ & $\begin{array}{l}\text { Definition for } \\
\text { cure or success }\end{array}$ & $N$ or $\%$ of success & $\begin{array}{c}N \text { or \% of } \\
\text { complications }\end{array}$ & $\begin{array}{l}N \text { or \% of } \\
\text { recurrence }\end{array}$ & $\begin{array}{l}\text { Testicular volume }(\mathrm{ml}) \text { or } \\
\text { testicular catch-up } \\
\text { growth }>9 \text { mo }(n)\end{array}$ & $\begin{array}{l}N \text { of pain } \\
\text { resolution }\end{array}$ & Sperm parameters & $\begin{array}{l}\text { Authors' } \\
\text { conclusions }\end{array}$ \\
\hline $\begin{array}{l}\text { Shiraishi et al (2016) } \\
{[12], 2008-2014}\end{array}$ & $24-85$ & $\begin{array}{l}\text { Resolution and } \\
\text { catch-up growth }\end{array}$ & $100 \%$ & $1 / 81$ & 0 & $\begin{array}{l}\text { Catch-up growth: } \\
\text { Subinguinal: } 70 \%(29 / 41) \\
\text { Inguinal: } 78 \%(31 / 40) \\
(p>0.05) \\
\text { Preop/postop vol. }(\mathrm{ml}) \\
\text { measured by orchidometer } \\
\text { and US: } \\
\text { Subinguinal: } 7.0 / 12.6 \\
\text { Inguinal: } 7.2 / 12.7\end{array}$ & $\begin{array}{l}\text { Scrotal pain } \\
\text { diminished } \\
\text { in } 24 / 24 \\
(100 \%) \\
3 \text { new-onset } \\
\text { scrotal pain in } \\
\text { subinguinal } \\
\text { group }\end{array}$ & NR & $\begin{array}{l}\text { Similar success, but } \\
\text { high inguinal is } \\
\text { easier: less veins, } \\
\text { larger artery }\end{array}$ \\
\hline $\begin{array}{l}\text { Spinelli et al (2016) } \\
\text { [13], 2008-2013 }\end{array}$ & 12 & $\begin{array}{l}\text { Resolution and } \\
\text { catch-up growth }\end{array}$ & $69 / 70(98.5)$ & 0 & 3 & $\begin{array}{l}\text { Numbers of catch-up } \\
\text { growth for hypotrophic } \\
\text { testis: } 39 / 70 \text { measured by } \\
\text { US }\end{array}$ & NR & NR & $\begin{array}{l}\text { Lymphatic and artery } \\
\text { sparing } \\
\text { varicocelectomy by } \\
\text { delivering testis and } \\
\text { ligating all collaterals } \\
\text { results in higher } \\
\text { catch-up growth }\end{array}$ \\
\hline $\begin{array}{l}\text { Yamamoto et al } \\
\text { (1995) [14], NR }\end{array}$ & 12 & $\begin{array}{l}\text { Catch-up growth, } \\
\text { semen analysis, } \\
\text { and hormones }\end{array}$ & NR & NR & NR & $\begin{array}{l}\text { Catch-up growth, preop/ } \\
\text { postop vol. }(\mathrm{ml}) \text { measured } \\
\text { by orchidometer: } \\
\text { Treated: } 21.0 / 25.2 \\
\text { Untreated: } 21.0 / 21.5 \\
\text { Control: } 24.6 / 24.9 \\
(p<0.03)\end{array}$ & NR & $\begin{array}{l}\text { Preop/postop sperm } \\
\text { conc } 10^{5}: \\
\text { Treated: } 50.5 / 73.9 \\
\text { Untreated: } 49.2 / 46.3 \\
(p<0.002) \\
\text { Control: } 50.2 / 53.1 \\
\text { Other parameters } \\
\text { comparable }\end{array}$ & $\begin{array}{l}\text { Varicocele treatment } \\
\text { in adolescents leads } \\
\text { to increased testis } \\
\text { volume and a higher } \\
\text { sperm concentration; } \\
\text { however, whether } \\
\text { early treatment will } \\
\text { improve testicular } \\
\text { function remains to } \\
\text { be elucidated }\end{array}$ \\
\hline $\begin{array}{l}\text { Zampieri et al (2007) } \\
{[15], 1999-2003}\end{array}$ & 18 & $\begin{array}{l}\text { Doppler } \\
\text { velocimetry after } \\
3 \text { mo postop }\end{array}$ & $116 / 122(95 \%)$ & $9 / 122$ & $5 / 122$ & NR & NR & $\begin{array}{l}\text { Only postop sperm } \\
\text { parameters available: } \\
\text { Patients with artery } \\
\text { preservation showed } \\
\text { better results for all } \\
\text { parameters }\end{array}$ & $\begin{array}{l}\text { Preservation of the } \\
\text { testicular artery Was } \\
\text { the best possible } \\
\text { option in terms of } \\
\text { semen quality }\end{array}$ \\
\hline $\begin{array}{l}\text { Mohseni et al (2011) } \\
\text { [16], 2006-2009 }\end{array}$ & $\begin{array}{l}15,12 \text {, and } \\
14 \text { mo for } \\
\text { groups } 1 \\
2 a \text {, and } 2 b \text {, } \\
\text { respectively }\end{array}$ & $\begin{array}{l}\text { Assessing for } \\
\text { testicular size by } \\
\text { US and recurrence } \\
\text { of varicocele }\end{array}$ & 69/74 (93.2\%) & NR & $5 / 74$ & $\begin{array}{l}\text { New-onset testicular } \\
\text { asymmetry in } 8 \text { of } \\
\text { untreated patients, } \\
\text { measured by US } \\
\text { Numbers of catch-up } \\
\text { growth for hypotrophic } \\
\text { testis: } 32 / 74\end{array}$ & NR & NR & $\begin{array}{l}\text { Shunt-type } \\
\text { varicocele is } \\
\text { associated with a } \\
\text { higher risk of } \\
\text { testicular hypotrophy } \\
\text { among untreated } \\
\text { patients }\end{array}$ \\
\hline
\end{tabular}

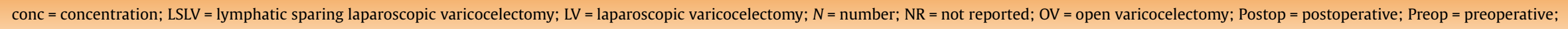
$\mathrm{RCT}$ = randomized controlled trial; SIL-V = single-incision laparoscopic varicocelectomy; US = ultrasonography; VC = varicocelectomy; vol. = volume. 


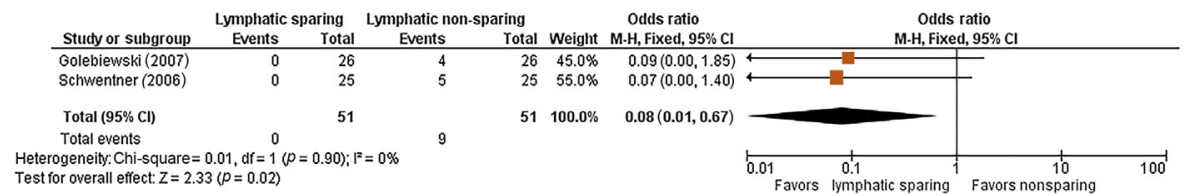

Fig. 3 - Forest plot demonstrating hydrocele occurrence after lymphatic sparing versus non-sparing surgery. $\mathrm{CI}=$ confidence interval; $\mathrm{M}$ - $\mathrm{H}=\mathbf{M a n t e l}$ Haenszel.

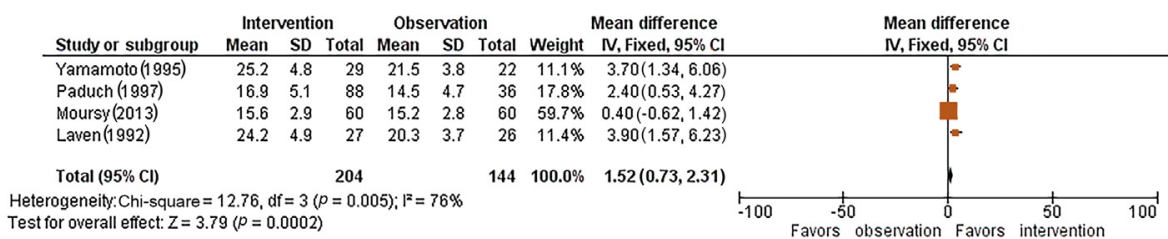

Fig. 4 - Forest plot demonstrating mean testicular volumes $(\mathrm{ml})$ for intervention versus observation groups. $\mathrm{CI}=$ confidence interval; $\mathrm{IV}=$ inverse variance; $\mathrm{SD}=$ standard deviation.

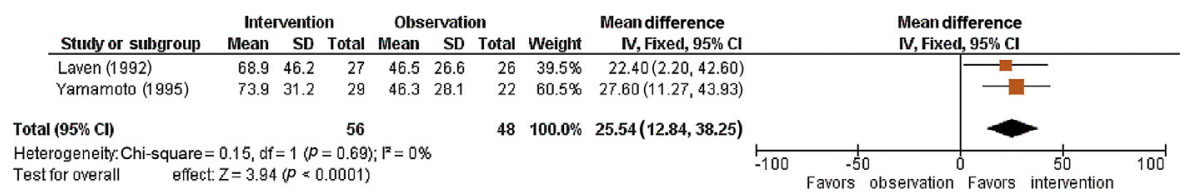

Fig. 5 - Forest plot demonstrating total sperm counts for intervention versus observation groups. $\mathrm{CI}=\mathrm{confidence}$ interval; $\mathrm{IV}=$ inverse variance; SD = standard deviation.

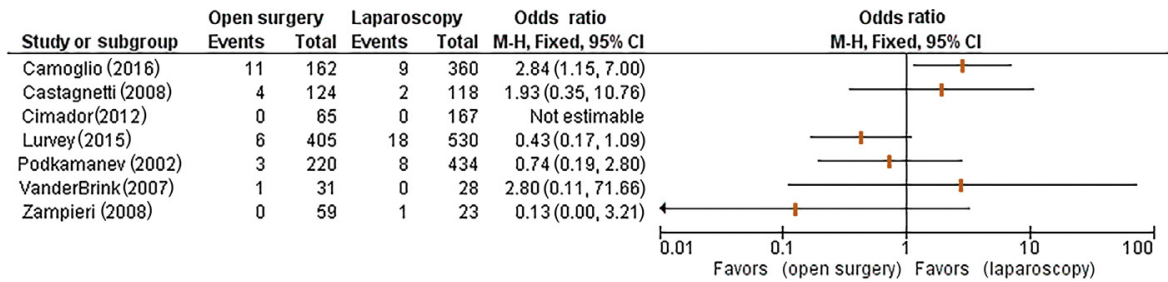

Fig. 6 - Forest plot demonstrating recurrence of open versus laparoscopic treatment of varicocele. $\mathrm{CI}=$ confidence interval; $\mathrm{M}$ - $\mathrm{H}=\mathrm{Mantel}-\mathrm{Haenszel}$.

Only one RCT and six NRSs compared the outcomes of open surgery and laparoscopy (Fig. 6). Six of the open surgeries were microscopic, two with loupe magnification and two were unclear in terms of the use of magnifiers. In addition, the type of surgery (subinguinal, Palomo, etc) is also unclear in the majority of the reported studies. The OR ranged from 0.13 to 2.84 . As a result, it remains unclear whether open or laparoscopic surgery is more successful for varicocele treatment. In addition, due to the lack of comparative data we were not able to discern a statistical difference in treatment success among the various forms of open surgery.

3.4.2.2. Complication of surgery/interventions. The complications reported by the included NRSs and case series included hydrocele, atrophy, wound infection, hematomas, scrotal emphysema, epididymitis, and shoulder pain. The most commonly reported complication was hydrocele, with a range of $0-29 \%$.
When looking into the NRSs, in parallel with the RCT outcomes, the number of hydrocele complications was decreased by performing lymphatic sparing surgery in the reported series $[27,28,36,41,47,49,50,60]$.

3.4.2.3. Testicular volume changes and catch-up growth. Testicular volume measurements and/or catch-up growth rates were recorded in 22 of the NRSs, whereas it was reported in 17 case series. Testicular catch-up growth rates after interventional varicocele treatment (antegrade sclerotherapy, retrograde embolization, etc.) were between $86 \%$ and $100 \%$ [26,93]. Following laparoscopic varicocelectomy, it was reported between $77 \%$ and $100 \%$ [20,31], whereas following open varicocelectomy (subinguinal, inguinal, Palomo, microscopic, etc) it was between $62.8 \%$ and $97.1 \%$ [36,68].

3.4.2.4. Changes in sperm parameters. Sperm parameters of the patients were available only in six of the NRSs, whereas it was 


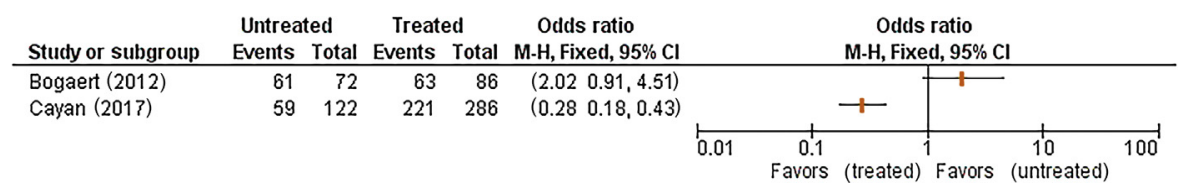

Fig. 7 - Forest plot demonstrating paternity of treated versus nontreated patients with varicocele. $\mathrm{CI}=$ confidence interval; M-H = Mantel-Haenszel.

reported in six case series. In NRSs, it was demonstrated that all parameters were increased after interventional, microscopic, and laparoscopic treatments $[26,47,48,58,61,62]$, with a mean follow-up between 17.6 mo and $10.6 \mathrm{yr}$. In the case series, where semen parameters were evaluated both before and after varicocelectomy, the sperm count, motility, and morphology significantly improved $[91,96,98]$.

3.4.2.5. Resolution of pain. Postoperative pain status (persistence/resolution) as an outcome of surgery/intervention was reported in three of the NRSs [18,26,53] and only in four case series [64,77,87,101]. Keene and Cervellione [26] reported outcomes of different techniques of antegrade sclerotherapy in 91 patients with G2 and G3 varicocele. Resolution of pain was observed in all 38 cases $(100 \%)$ who had pain preoperatively. In another case series by Poddoubny et al. [77], laparoscopic varicocelectomy was performed in 180 children with left-sided varicocele. Out of 28 children who had pain preoperatively, 26 had resolution of pain (92.9\%).

3.4.2.6. Hormonal status. Hormonal status including LH, FSH, and testosterone was recorded in three of the NRSs $[38,47,48]$ and in only one case series [83]. In two of the NRSs [38,48], pre- and postoperative hormonal levels were found comparable; however, in the study by Çayan et al. [47], mean testosterone levels were found to be elevated (3.61-4.96 ng/ml) after microscopic subinguinal or inguinal varicocelectomy.

3.4.2.7. Paternity rates. Two NRSs [19,47] and two case series $[96,100]$ reported paternity rates. Pajovich and Radojevic [96] reported long-term outcomes of laparoscopic varicocelectomy, and they found $75 \%$ paternity rate $(12 / 16)$ in the study population. Salzhauer et al. [100] found $100 \%$ paternity rate after varicocelectomy (Ivanissevich and Palomo techniques) in 43 patients who responded the questionnaire. Both Bogaert et al. [19] and Çayan et al. [47] compared the outcomes of treatment versus observation. Bogaert et al. [19] found 78\% paternity rate in patients who underwent antegrade sclerotherapy whereas $85 \%$ paternity rate in controls, most of their patients did not have testicular hypotrophy. They concluded that varicocele intervention does not improve the paternity rate. However, in the study by Çayan et al. [47], 286 patients underwent microsurgical varicocelectomy and 122 were observed. All patients had testicular hypotrophy and $>50 \%$ of the cases underwent bilateral varicocelectomy. Paternity rate was $77.3 \%$ in the treated group versus $48.4 \%$ in the untreated group, favoring treatment in adolescent varico- cele. Forest plot demonstration of paternity is provided in Figure 7.

\subsection{Discussion}

\subsubsection{Principal findings}

Our SR and MA provided the available evidence in the literature on the benefits and harms of varicocele treatment in children and adolescents, which are mentioned below.

Success rates (disappearance of varicocele) were $>85 \%$ among all included studies. Owing to a lack of RCTs, we could not identify a surgical technique that was superior to the others. We found that open surgery and laparoscopy may have similar treatment success.

An MA based on available RCTs revealed improved testicular volume and increased total sperm concentration in children who underwent intervention compared with observation. The most common complication reported was hydrocele. A significant decrease in hydrocele formation was observed in lymphatic sparing versus non-lymphatic sparing surgery.

Resolution of pain was another parameter investigated in our study. Although reporting this outcome was extremely rare in the included studies, all included studies reported $>90 \%$ of pain resolution.

Very few studies investigated the hormonal status of patients and the majority of them revealed that hormonal levels were within normal ranges both before and after treatment. We presume that a future research on subfertile patients would provide meaningful outcomes.

Paternity is one of the most important outcomes after varicocelectomy, but in contrast, it is the least reported outcome in the literature due to the necessity of long-term follow-up. Both comparative studies were biased by several factors and the outcome was conflicting. In the study by Bogaert et al. [19], only 361 of the 661 included patients completed the survey regarding the paternity rate. In the study by Çayan et al. [47], the follow-up time was significantly higher in the treated group versus the nontreated group. Based on the available data, the effect of surgery on paternity rate cannot be determined.

\subsubsection{Implications for clinical practice}

With this SR and MA, the benefits of varicocele treatment in children and adolescents are demonstrated in terms of improved testicular volume and sperm parameters. At this moment, we are not able to recommend any surgical/ interventional technique as the standard treatment of varicocele. Lymphatic preservation is highly recommended in order to decrease the rates of hydrocele. Paternity and 
fertility issues still remain unclear. We could not identify an ideal candidate for varicocele treatment according to the available literature. However, adolescents with high-grade varicocele, hypotrophic left testicle, pain, and poor sperm parameters would be more likely to benefit from varicocele treatment.

\subsubsection{Further research}

Undoubtedly, RCTs are required to elucidate which surgical/ interventional technique is the best option in treating varicocele. Long-term outcomes such as paternity and fertility still need to be studied. There is no randomized trial comparing paternity rates of intervention/surgery versus observation. Additionally, RCTs (treatment vs no treatment, open surgery vs laparoscopy, artery sparing vs non-artery sparing, etc.) providing pre- and postoperative semen parameters are still not available in the literature. Optimization of the diagnostic parameters of varicocele and uniform definition of the treatment success is required for further investigation about varicocele. The definition of "catch-up growth" should also be uniform among the future studies. Finally, the utility of studying hormonal status in otherwise healthy men with varicocele is questionable.

\subsubsection{Limitations and strengths}

Our findings are limited by the heterogeneity of the type of the interventions and the lack of long-term data demonstrating sperm parameters and paternity rate. Moderate evidence exists on the benefits of varicocele treatment in terms of testicular volume and sperm concentration. Another important limitation of our study was that the definitions were highly variable among the included studies. Indications for surgery, grading of varicocele, treatment success, and some complications were not uniformly reported. Catch-up growth and testicular hypotrophy were also other parameters that lack standardized definitions in the literature.

Pubertal boys and adolescents are growing up individuals and their genitalia are in a continuous developmental stage, so that comparison among them is difficult. There are no normal values of spermiogram, and this is another important limitation.

Although we could not demonstrate the effects of varicocele treatment on long-term outcomes such as paternity and fertility, we were able to provide an overview of the best available evidence. With this up-to-date SR and MA including 98 eligible studies with 16130 patients, we were able to meta-analyze many parameters including testicular volume changes, spermiogram outcomes, and benefits of lymphatic sparing surgeries. We could also demonstrate forest plot tables of the comparison of laparoscopy and open surgery, and furthermore paternity rates in the long term. However, these parameters were not meta-analyzed due to a lack of RCTs and in order to avoid any misinterpretation of the outcomes.

This SR was performed by a group of experts including clinicians and methodologists (EAU Pediatric Urology Guideline Panel) according to PRISMA guidelines, and the results will be incorporated into the 2019 practice guidelines.

\section{Conclusions}

According to the contemporary evidence, which is supported by a systematical assessment and MA, the benefits of varicocele correction in children and adolescents when compared with observation are increases in testicular volume and sperm concentration. Lymphatic sparing surgery is superior to nonsparing surgery in terms of decreasing hydrocele occurrence postoperatively. In addition, current evidence does not demonstrate superiority of any surgical/interventional technique regarding treatment success. Finally, long-term outcomes including paternity and fertility still remain unknown.

This SR was performed under the auspices of the European Association of Urology and the European Society for Paediatric Urology, Pediatric Urology Guidelines Panel.

Author contributions: Mesrur Selcuk Silay had full access to all the data in the study and takes responsibility for the integrity of the data and the accuracy of the data analysis.

Study concept and design: Silay, Undre, Quadackaers, Hoen, Dogan, Kocvara, Nijman, Stein, Tekgul, Radmayr.

Acquisition of data: Silay, Undre, Quadackaers, Hoen.

Analysis and interpretation of data: Silay, Undre, Quadackaers, Hoen.

Drafting of the manuscript: Silay, Undre, Quadackaers, Hoen.

Critical revision of the manuscript for important intellectual content: Dogan, Bogaert, Kocvara, Nijman, Radmayr.

Statistical analysis: Hoen, Silay.

Obtaining funding: None.

Administrative, technical, or material support: None.

Supervision: Tekgul, Stein, Bogaert.

Other: None.

Financial disclosures: Mesrur Selcuk Silay certifies that all conflicts of interest, including specific financial interests and relationships and affiliations relevant to the subject matter or materials discussed in the manuscript (eg, employment/affiliation, grants or funding, consultancies, honoraria, stock ownership or options, expert testimony, royalties, or patents filed, received, or pending), are the following: None.

Funding/Support and role of the sponsor: one.

\section{Appendix A. Supplementary data}

Supplementary data associated with this article can be found, in the online version, at https://doi.org/10.1016/j. eururo.2018.09.042.

\section{References}

[1] EAU/ESPU guidelines on pediatric urology in EAU guidelines. Paper presented at: The EAU Annual Congress Copenhagen; March 1618; Copenhagen. 2018.

[2] Nork JJ, Berger JH, Crain DS, Christman MS. Youth varicocele and varicocele treatment: a meta-analysis of semen outcomes. Fertil Steril 2014;102:381-7.

[3] Zhou T, Zhang W, Chen Q, et al. Effect of varicocelectomy on testis volume and semen parameters in adolescents: a meta-analysis. Asian J Androl 2015;17:1012-6. 
[4] Locke JA, Maryam N, Kourosh A. Treatment of varicocele in children and adolescents: a systematic review and meta-analysis of randomized controlled trials. J Pediatr Urol 2017;13:437-45.

[5] Laven JS, Haans LC, Mali WP, te Velde ER, Wensing CJ, Eimers JM. Effects of varicocele treatment in adolescents: a randomized study. Fertil Steril 1992;58:756-62.

[6] Podkamenev VV, Stalmakhovich VN, Urkov PS, Solojev AA, Iljin VP. Laparoscopic surgery for pediatric varicoceles: randomized controlled trial. J Pediatr Surg 2002;37:727-9.

[7] Golebiewski A, Krolak M, Komasara L, Czauderna P. Dye-assisted lymph vessels sparing laparoscopic varicocelectomy. J Laparoendosc Adv Surg Tech A 2007; 17:360-3.

[8] Marte A, Pintozzi L, Cavaiuolo S, Parmeggiani P. Single-incision laparoscopic surgery and conventional laparoscopic treatment of varicocele in adolescents: comparison between two techniques. Afr J Pediatr Surg 2014;11:201-5.

[9] Moursy EE, ElDahshoury MZ, Hussein MM, Mourad MZ, Badawy AA. Dilemma of adolescent varicocele: long-term outcome in patients managed surgically and in patients managed expectantly. J Pediatr Urol 2013;9:1018-22.

[10] Paduch DA, Niedzielski J. Repair versus observation in adolescent varicocele: a prospective study. J Urol 1997;12:410-3.

[11] Schwentner C, Radmayr C, Lunacek A, et al. Laparoscopic varicocele ligation in children and adolescents using isosulphan blue: a prospective randomized trial. BJU Int 2006;98:861-5.

[12] Shiraishi K, Oka S, Matsuyama H. Surgical comparison of subinguinal and high inguinal microsurgical varicocelectomy for adolescent varicocele. Int J Urol 2016;23:338-42.

[13] Spinelli C, Strambi S, Busetto M, et al. Microsurgical inguinal varicocelectomy in adolescents: delivered versus not delivered testis procedure. Can J Urol 2016;23:8254-9.

[14] Yamamoto M, Hibi H, Katsuno S, Miyake K. Effects of varicocelectomy on testis volume and semen parameters in adolescents: a randomized prospective study. Nagoya J Med Sci 1995;58:127-32.

[15] Zampieri N, Zuin V, Corroppolo M, Chironi C, Cervellione RM, Camoglio FS. Varicocele and adolescents: semen quality after 2 different laparoscopic procedures. J Androl 2007;28:727-33.

[16] Mohseni MJ1, Nazari H, Amini E, et al. Shunt-type and stop-type varicocele in adolescents: prognostic value of these two different hemodynamic patterns. Fertil Steril 2011;96:1091-6.

[17] Belloli G, D’Agostino S, Musi L, Campobasso P. Adolescent varicocele: operative anatomy and tricks for successful correction. Eur J Pediatr Surg 1995;5:219-21.

[18] Beutner S, May M, Hoschke B, et al. Treatment of varicocele with reference to age: a retrospective comparison of three minimally invasive procedures. Surg Endosc 2007;21:61-5.

[19] Bogaert G, Orye C, De Win G. Pubertal screening and treatment for varicocele do not improve chance of paternity as adult. J Urol 2013;189:2298-303.

[20] Castagnetti M, Cimador M, DiPace MR, Catalano P, DeGrazia E. 'Preemptive hydrocelectomy' in subinguinal varicocelectomy. Urol Int 2008;81:14-6.

[21] Castagnetti M, Cimador M, Catalano P, DiPace M, Sergio M, De Grazia E. Evolving management of adolescent varicocele. J Pediatr Urol 2008;4:107-12.

[22] Cimador M, Di Pace MR, Peritore M, Sergio M, Castagnetti M, De Grazia E. The role of Doppler ultrasonography in determining the proper surgical approach to the management of varicocele in children and adolescents. BJU Int 2006;97:1291-7.

[23] D’Alessio A, Piro E, Beretta F, Brugnoni M, Marinoni F, Abati L. Lymphatic preservation using methylene blue dye during varicocele surgery: a single-center retrospective study. J Pediatr Urol 2008;4:138-40.
[24] Esposito C, Iaquinto M, Escolino M, et al. Technical standardization of laparoscopic lymphatic sparing varicocelectomy in children using isosulfan blue. J Pediatr Surg 2014;49:660-3.

[25] Fast AM, Deibert CM, Van Batavia JP, Nees SN, Glassberg KI. Adolescent varicocelectomy: does artery sparing influence recurrence rate and/or catch-up growth? Andrology 2014;2:159-64.

[26] Keene DJB, Cervellione RM. Antegrade sclerotherapy in adolescent varicocele patients. J Pediatr Urol 2017;13:305, e1-e6.

[27] Kocvara R, Dolezal J, Hampl R, et al. Division of lymphatic vessels at varicocelectomy leads to testicular oedema and decline in testicular function according to the LH-RH analogue stimulation test. Eur Urol 2003;43:430-5

[28] Kocvara R, Dvorácek J, Sedlácek J, Díte Z, Novák K. Lymphatic sparing laparoscopic varicocelectomy: a microsurgical repair. J Urol 2005;173:1751-4.

[29] Lund L, Tang YC, Roebuck D, Lee KH, Liu K, Yeung CK. Testicular catch-up growth after varicocele correction in adolescents. Pediatr Surg Int 1999;15:234-7.

[30] Lurvey R, Durbin-Johnson B, Kurzrock EA. Adolescent varicocele: a large multicenter analysis of complications and recurrence in academic programs. J Pediatr Urol 2015;11:186, e1-e6.

[31] Mancini S, Bulotta AL, Molinaro F, Ferrara F, Tommasino G, Messina M. Surgical retroperitoneoscopic and transperitoneoscopic access in varicocelectomy: duplex scan results in pediatric population. J Pediatr Urol 2014;10:1037-42.

[32] Messina M, Zagordo L, Di Maggio G, Molinaro F, Abate V, Nardi N. Testicular hypotrophy in varicocele: pre and postoperative echographic follow-up in the pediatric age. Minerva Urol Nefrol 2006;58:151-5.

[33] Misseri R, Gershbein AB, Horowitz M, Glassberg KI. The adolescent varicocele. II: the incidence of hydrocele and delayed recurrent varicocele after varicocelectomy in a long-term follow-up. BJU Int 2001;87:494-8.

[34] Nees SN, Glassberg KI. Observations on hydroceles following adolescent varicocelectomy. J Urol 2011;186:2402-7.

[35] Palmer LS, Maizels M, Kaplan WE, Stokes S, Firlit CF. The influence of surgical approach and intraoperative venography on successful varicocelectomy in adolescents. J Urol 1997;158:1201-4.

[36] Poon SA, Kozakowski KA, Decastro GJ, Gjertson CK, Glassberg KI. Adolescent varicocelectomy: postoperative catch-up growth is not secondary to lymphatic ligation. J Pediatr Urol 2009;5:37-41.

[37] Reitelman C, Burbige KA, Sawczuk IS, Hensle TW. Diagnosis and surgical correction of the pediatric varicocele. J Urol 1987;138:1038-40.

[38] Silveri M, Bassani F, Adorisio O. Changing concepts in microsurgical pediatric varicocelectomy: is retroperitoneal approach better than subinguinal one? Urol J 2015;12:2032-5.

[39] Spinelli C, Di Giacomo M, Lo Piccolo R, Martin A, Messineo A. The role of testicular volume in adolescents with varicocele: the better way and time of surgical treatment. J Urol 2010;184:1722-6.

[40] Tsikopoulos GK, Roussis XS, Georgakis J, Papouis G, Tryfonas GJ. Does incision of the tunica vaginalis during the Palomo procedure prevent post-operative hydrocele? Br J Urol 1998;81:612-3.

[41] VanderBrink BA, Palmer LS, Gitlin J, Levitt SB, Franco I. Lymphaticsparing laparoscopic varicocelectomy versus microscopic varicocelectomy: is there a difference? Urology 2007;70:1207-10.

[42] Zampieri N, Corroppolo M, Zuin V, Cervellione RM, Ottolenghi A, Camoglio FS. Longitudinal study of semen quality in adolescents with varicocele: to treat or not? Urology 2007;70:989-93.

[43] Atassi O, Kass EJ, Steinert BW. Testicular growth after successful varicocele correction in adolescents: comparison of artery sparing techniques with the Palomo procedure. J Urol 1995:153:482-3. 
[44] Bansal D, Riachy E, Defoor Jr WR, et al. Pediatric varicocelectomy: a comparative study of conventional laparoscopic and laparoendoscopic single-site approaches. J Endourol 2014;28:513-6.

[45] Bertschy C, Liard A, Bawab F, Bachy B, Le Dosseur P, Mitrofanoff P. Idiopathic varicocele in children and adolescents-which therapeutic choice? Eur J Pediatr Surg 1997;7:341-4.

[46] Camoglio FS, Zampieri N. Varicocele treatment in paediatric age: relationship between type of vein reflux, surgical technique used and outcomes. Andrologia 2016;48:389-92.

[47] Çayan S, Şahin S, Akbay E. Paternity rates and time to conception in adolescents with varicocele undergoing microsurgical varicocele repair vs observation only: a single institution experience with 408 patients. J Urol 2017;198:195-201.

[48] Çayan S, Acar D, Ulger S, Akbay E. Adolescent varicocele repair: long-term results and comparison of surgical techniques according to optical magnification use in 100 cases at a single university hospital. J Urol 2005;174:2003-6.

[49] Chiarenza SF, Giurin I, Costa L, et al. Blue patent lymphography prevents hydrocele after laparoscopic varicocelectomy: 10 years of experience. Laparoendosc Adv Surg Tech A 2012;22:930-3.

[50] Choi CI, Park KC, Lee TH, Hong YK. Recurrence rates in pediatric patients undergoing microsurgical subinguinal varicocelectomy with and without testicular delivery. J Pediatr Surg 2017;52:150710.

[51] Cimador M, Pensabene M, Sergio M, Caruso AM, De Grazia E. Focus on paediatric and adolescent varicocoele: a single institution experience. Int J Androl 2012;35:700-5.

[52] Kass EJ, Marcol B. Results of varicocele surgery in adolescents: a comparison of techniques. J Urol 1992;148:694-6.

[53] Lebed B, Packer M, Husmann D, Zaontz M. Results and complications of adolescent varicocele repair with intraoperative sodium morrhuate sclerotherapy. J Urol 1998;180:1837-41.

[54] Mazzoni G, Spagnoli A, Lucchetti MC, Villa M, Capitanucci ML, Ferro F. Adolescent varicocele: Tauber antegrade sclerotherapy versus Palomo repair. J Urol 2001;166:1462-4.

[55] Mazzoni G, Fiocca G, Minucci S, et al. Varicocele: a multidisciplinary approach in children and adolescents. J Urol 1999;162:1757-78.

[56] Pintus C, Rodriguez Matas MJ, Manzoni C, Nanni L, Perrelli L. Varicocele in pediatric patients: comparative assessment of different therapeutic approaches. Urology 2001;57:154-7.

[57] Polok M, Patkowski D, Apoznański W, Dorobisz U, Laska E, Chrzan R. Electrocoagulation versus clips in laparoscopic varicocelectomy in boys. J Laparoendosc Adv Surg Tech A 2010;20:277-80.

[58] Pozza D, Gregori A, Ossanna P, Amodeo S, Malizia S, Marchionni L. Is it useful to operate on adolescent patients affected by left varicocele? J Androl 1994;15:43-6.

[59] Riccabona M, Oswald J, Koen M, Lusuardi L, Radmayr C, Bartsch G. Optimizing the operative treatment of boys with varicocele: sequential comparison of 4 techniques. J Urol 2003;169:666-8.

[60] Rizkala E, Fishman A, Gitlin J, Zelkovic P, Franco I. Long term outcomes of lymphatic sparing laparoscopic varicocelectomy. J Pediatr Urol 2013;9:458-63.

[61] Yu W, Rao T, Ruan Y, Yuan R, Cheng F. Laparoscopic varicocelectomy in adolescents: artery ligation and artery preservation. Urology 2016;89:150-4.

[62] Zampieri N, Cervellione RM. Varicocele in adolescents: a 6-year longitudinal and followup observational study. J Urol 2008;180:1653-6.

[63] Zampieri N, Mantovani A, Ottolenghi A, Camoglio FS. Testicular catch-up growth after varicocelectomy: does surgical technique make a difference? Urology 2009;73:289-92.

[64] Belloli G, D’Agostino S, Zen F, Ioverno E. Fertility rates after successful correction of varicocele in adolescence and adulthood. Eur J Pediatr Surg 1995;5:216-8.
[65] Camoglio FS, Cervellione RM, Bruno C, et al. Microsurgical spermatico-epigastric venous anastomosis in the treatment of varicocele in children: assessment of long-term patency. Eur J Pediatr Surg 2003;13:256-9.

[66] Chrouser K, Vandersteen D, Crocker J, Reinberg Y. Nerve injury after laparoscopic varicocelectomy. J Urol 2004;172:691-3.

[67] Cobellis G, Mastroianni L, Cruccetti A, Amici G, Martino A. Retroperitoneoscopic varicocelectomy in children and adolescents. J Pediatr Surg 2005;40:846-9.

[68] Feber KM, Kass EJ. Varicocelectomy in adolescent boys: long-term experience with the Palomo procedure. J Urol 2008;180:1657-9.

[69] Galfano A, Novara G, Iafrate M, et al. Surgical outcomes after modified antegrade scrotal sclerotherapy: a prospective analysis of 700 consecutive patients with idiopathic varicocele. J Urol 2008; 179:1933-7.

[70] Gill B, Kogan SJ, Maldonado J, Reda E, Levitt SB. Significance of intraoperative venographic patterns on the postoperative recurrence and surgical incision placement of pediatric varicoceles. J Urol 1990;144:502-5.

[71] Granata C, Oddone M, Toma P, Mattioli G. Retrograde percutaneous sclerotherapy of left idiopathic varicocele in children: results and follow-up. Pediatr Surg Int 2008;24:583-7.

[72] Hassan JM, Adams MC, Pope 4th JC, Demarco RT, Brock 3rd JW. Hydrocele formation following laparoscopic varicocelectomy. J Urol 2006;175:1076-9.

[73] Niedzielski J, Paduch DA. Recurrence of varicocele after high retroperitoneal repair: implications of intraoperative venography. J Urol 2001;165:937-40.

[74] Nyirády P, Kiss A, Pirót L, et al. Evaluation of 100 laparoscopic varicocele operations with preservation of testicular artery and ligation of collateral vein in children and adolescents. Eur Urol 2002;42:594-7.

[75] Nyirády P, Pirót L, Altorjay A, et al. Laparoscopic varicocele operation: a chance to prevent the recurrence. Acta Chir Hung 1998;37:201-4.

[76] Paradiso FV, Mason EJ, Nanni L. Antegrade sclerotherapy to treat all types of varicoceles in the pediatric population: experience of a single center. Urology 2016;98:149-53.

[77] Poddoubny IV, Dronov AF, Kovarski SL, Korznikova IN, Darenkov IA, Zalikhin DV. Laparoscopic ligation of testicular veins for varicocele in children. A report of 180 cases. Surg Endosc 2000;14:1107-9.

[78] Reyes BL, Trerotola SO, Venbrux AC, et al. Percutaneous embolotherapy of adolescent varicocele: results and long-term followup. J Vasc Interv Radiol 1994;5:131-4.

[79] Schiff J, Kelly C, Goldstein M, Schlegel P, Poppas D. Managing varicoceles in children: results with microsurgical varicocelectomy. BJU Int 2005;95:399-402.

[80] Urbanowicz W, Dobrowolska-Glazar B, Wolnicki M, Honkisz I. Results of varicocele treatment with laparoscopic Palomo technique in children and adolescents in years 2002-2012. Przegl Lek 2014;71:415-7.

[81] Varlet F, Becmeur F, Groupe d'Etudes en Coeliochirurgie Infantile. Laparoscopic treatment of varicoceles in children. Multicentric prospective study of 90 cases. Eur J Pediatr Surg 2001;11:399-403.

[82] Zaupa P, Mayr J, Höllwarth ME. Antegrade scrotal sclerotherapy for treating primary varicocele in children. BJU Int 2006;97:809-12.

[83] Bryniarski P, Kaletka Z, Huk J, et al. Testicular volume and fertility potential in men operated due to varicocele and testicular hypotrophy in adolescence. Cent Eur J Urol 2013;66:56-9.

[84] Campobasso P. Blue venography in adolescent varicocelectomy: a modified surgical approach. J Pediatr Surg 1997;32:1298-301.

[85] Diamond DA, Xuewu J, Cilento Jr BG, et al. Varicocele surgery: a decade's experience at a children's hospital. BJU Int 2009;104:246-9. 
[86] Esposito C, Monguzzi G, Gonzalez-Sabin MA, et al. Results and complications of laparoscopic surgery for pediatric varicocele. J Pediatr Surg 2001;36:767-9.

[87] Fayad F, Sellier N, Chabaud M, et al. Percutaneous retrograde endovascular occlusion for pediatric varicocele. J Pediatr Surg 2011;46:525-9.

[88] Greenfield SP, Seville P, Wan J. Experience with varicoceles in children and young adults. J Urol 2002;168:1684-8.

[89] Kim KS, Lee C, Song SH, et al. Impact of internal spermatic artery preservation during laparoscopic varicocelectomy on recurrence and the catch-up growth rate in adolescents. J Pediatr Urol 2014;10:435-40.

[90] Koyle MA, Oottamasathien S, Barqawi A, Rajimwale A, Furness 3rd PD. Laparoscopic Palomo varicocele ligation in children and adolescents: results of 103 cases. J Urol 2004;172:1749-52.

[91] Ku JH, Kim SW, Park K, Paick JS. Benefits of microsurgical repair of adolescent varicocele: comparison of semen parameters in fertile and infertile adults with varicocele. Urology 2005;65: 554-558.

[92] Mazzoni G. Adolescent varicocele: treatment by antegrade sclerotherapy. J Pediatr Surg 2001;36:1546-50.

[93] Mazzoni G, Fiocca G, Oriolo L, Calisti A. Varicocele in adolescence: indications for treatment and long-term follow-up. Ital J Pediatr 2006;32:273-5.
[94] Méndez-Gallart R, Bautista Casasnovas A, Estévez Martínez E, et al. Reactive hydrocele after laparoscopic Palomo varicocele ligation in pediatrics. Arch Esp Urol 2010;63:532-6.

[95] Nagar H, Mabjeesh NJ. Decision-making in pediatric varicocele surgery: use of color Doppler ultrasound. Pediatr Surg Int 2000;16:75-6.

[96] Pajovich B, Radojevic N. Prospective follow up of fertility after adolescent laparoscopic varicocelectomy. Eur Rev Med Pharmacol Sci 2013;17:1060-3.

[97] Pieri S, Agresti P, Morucci M, De'Medici L, Fiocca G, Calisti A. [A transbranchial approach for the percutaneous therapy of pediatric varicocele]. Radiol Med 2003;106:221-31.

[98] Pozza D, Gregori A, Pulone M. Surgical treatment of varicocele at adolescence. Acta Hung Surg 1994;34:263-6.

[99] Renaux-Petel M, Vivier PH, Comte D, Beurdeley M, Liard A, Bachy B. Idiopathic varicocele in adolescents: risks of the inguinal approach. Eur J Pediatr Surg 2013;23:285-8.

[100] Salzhauer EW, Sokol A, Glassberg KI. Paternity after adolescent varicocele repair. Pediatrics 2004;114:1631-3.

[101] Yaman O, Soygur T, Zumrutbas AE, Resorlu B. Results of microsurgical subinguinal varicocelectomy in children and adolescents. Urology 2006;68:410-2.

[102] Zampieri N, Chironi C, Sulpasso M. Treatment of varicocele with transfemoral retrograde sclero-embolization in pediatric patients under local anesthesia. Minerva Pediatr 2015;67:227-9. 